\title{
A study of tribendimidine effects in vitro and in vivo on the liver fluke Opisthorchis felineus
}

\author{
Maria Y. Pakharukova ${ }^{1,2^{*}}$, Vladimir A. Samsonov ${ }^{3}$, Elena A. Serbina ${ }^{4}$ and Viatcheslav A. Mordvinov ${ }^{1}$
}

\begin{abstract}
Background: The food-borne liver fluke Opisthorchis felineus is an epidemiologically important species and the causative agent of opisthorchiasis across an extensive territory of Eurasia. For decades, treatment of opisthorchiasis has been based on praziquantel. Tribendimidine could be an alternative drug that has been successfully tested for Opisthorchis viverrini and Clonorchis sinensis infections. We aimed to assess tribendimidine effects in comparison with praziquantel in vivo and in vitro against the liver fluke Opisthorchis felineus.

Results: In this study we (i) calculated half-maximal inhibitory concentrations $\left(I C_{50}\right)$ by motility tests against $O$. felineus adults and newly excysted metacercarie after tribendimidine treatment in vitro; (ii) determined whether tribendimidine and PZQ effects on adult liver flukes are dependent on or mediated by white blood cells; and (iii) tested in vivo the anthelmintic activity of tribendimidine on juvenile and adult worms. We found that the efficiency of tribendimidine in vitro was similar $\left(\mathrm{IC}_{50}=0.23 \mu \mathrm{M}\right.$ for newly excysted metacercariae and $0.19 \mu \mathrm{M}$ for adult worms) to that of praziquantel ( $\left(C_{50} 0.98 \mu \mathrm{M}\right.$ for newly excysted metacercariae and $0.47 \mu \mathrm{M}$ for adult worms). The treatment of adult worms in vivo with praziquantel or tribendimidine at $400 \mathrm{mg} / \mathrm{kg}$ resulted in a $76 \%$ and $77.2 \%$ reduction, respectively, in the worm burden during chronic infection.
\end{abstract}

Conclusions: The differences between WBR values after PZQ and TBN treatment were not significant, thus tribendimidine was as effective as praziquantel against $O$. felineus liver flukes. Given the broad-spectrum activity of tribendimidine and efficacy against $O$. felineus, this drug may be a promising candidate for the treatment of opisthorchiasis felinea and other liver fluke infections.

Keywords: Food-borne trematodes, Opisthorchis felineus, Tribendimidine, in vivo, in vitro, Hamster model

\section{Background}

The liver fluke Opisthorchis felineus is a member of the triad of epidemiologically important species of food-borne trematodes (O. felineus, O. viverrini and C. sinensis), and the causative agent of opisthorchiasis felinea over an extensive territory across Eurasia [1-3]. The prevalence of $O$. felineus infection in the population of the endemic regions of Western Siberia is 10-45\% [1, 2]. Human infection results from eating raw or undercooked freshwater cyprinoid fish carrying metacercariae of the parasite $[1,2]$. Metacercariae excyst in the duodenum, and the juvenile

\footnotetext{
*Correspondence: pmaria@yandex.ru

${ }^{1}$ Institute of Cytology and Genetics of the Siberian Branch of the Russian Academy of Sciences (SB RAS), 10 Lavrentiev Ave, Novosibirsk 630090, Russia

${ }^{2}$ Novosibirsk State University, 2 Pirogova Str, Novosibirsk 630090, Russia

Full list of author information is available at the end of the article
}

parasites ascend into the bile ducts, where development into adult worms proceeds over the course of four weeks. The adult liver flukes reside within the bile ducts and gall-bladder [2] and shed eggs that enter the gastrointestinal tract and are released with feces.

The clinical manifestations and pathology induced by chronic infection with $O$. felineus, $O$. viverrini and $C$. sinensis liver flukes are similar $[4,5]$. The liver flukes induce several hepatobiliary disorders, including hepatomegaly, cholangitis, periductal fibrosis, chronic inflammation, liver abscesses $[2,3,6]$ and potentially cholangiocarcinoma. The International Agency for Research on Cancer classifies the flukes of the family

(c) The Author(s). 2019 Open Access This article is distributed under the terms of the Creative Commons Attribution 4.0 International License (http://creativecommons.org/licenses/by/4.0/), which permits unrestricted use, distribution, and reproduction in any medium, provided you give appropriate credit to the original author(s) and the source, provide a link to the Creative Commons license, and indicate if changes were made. The Creative Commons Public Domain Dedication waiver (http://creativecommons.org/publicdomain/zero/1.0/) applies to the data made available in this article, unless otherwise stated. 
Opisthorchiidae (O. viverrini and $C$. sinensis) as group 1 agents and major risk factors for cholangiocarcinoma [4].

To date, the only drug of choice for the treatment of opisthorchiasis, clonorchiasis, schistosomiasis and other fluke infections has been praziquantel (PZQ). PZQ disrupts calcium ion homeostasis and antagonizes voltagegated calcium channels. Drug discovery for trematode infections has a high priority [7] to avoid the development of resistance to PZQ in the future. Studies on alternative drugs for the treatment of liver fluke infections have shown that tribendimidine (TBN) is a good candidate [8-10]. It is a symmetrical diamidine derivative of amidantel and was developed in China for use in humans in the mid-1980s [11]. TBN is a cholinergic agonist that is selective for nicotinic acetylcholine receptors. TBN has a broad-spectrum activity against intestinal nematodes. The efficiency of TBN against trematodes infection is variable $[9,12]$. The drug is effective against $O$. viverrini and $C$. sinensis infections, but ineffective against S. mansoni infection [9]. To our knowledge, TBN has never been tested against O. felineus infection.

The aims of the present study were to (i) test in vitro anthelmintic activity of $\mathrm{TBN}$ by calculating $\mathrm{IC}_{50}$ values via motility tests on juvenile and adult O. felineus worms; (ii) assess if TBN and PZQ effects on liver flukes are dependent on or mediated by white blood cells; and (iii) test in vivo the anthelmintic activity of TBN on juvenile and adult worms.

\section{Methods}

\section{Compounds}

TBN [(1E,1'E)- $\mathrm{N}^{\prime}, \mathrm{N}^{\prime \prime}-\left(4,4^{\prime}-\left(1 \mathrm{E}, 1^{\prime} \mathrm{E}\right)-(1,4-\right.$ phenylenebismethan-1-yl-1-ylidene))bis(azan-1-yl-1-ylidene)bis(4,1-p henylene))bis(N,N-dimethylacetimidamide)] was synthesized and provided by the Vorozhtsov Institute of Organic Chemistry of the Siberian Branch of the Russian Academy of Sciences (Novosibirsk, Russia). Spectroscopic characteristics confirmed the structure of the compound (purity 99\%).

For in vitro tests, PZQ (Sigma-Aldrich, St Louis, USA) and TBN were dissolved in dimethyl sulfoxide (DMSO) (Sigma-Aldrich) to obtain $1 \mathrm{mM}$ stock solutions.

For in vivo experiments, PZQ and TBN were prepared as a suspension in an aqueous solution of $7 \%$ Tween $80(\mathrm{v} / \mathrm{v})$ and $3 \%$ ethanol $(\mathrm{v} / \mathrm{v})$ before oral administration $(10 \mathrm{ml} / \mathrm{kg})$ and were administered at $400 \mathrm{mg} / \mathrm{kg}$ body weight.

\section{Animals and infection}

Syrian hamsters (Mesocricetus auratus) were purchased from the Animal Facility of the ICG SB RAS. Before infection, the animals were allowed to acclimate for one week at our animal facility. They were kept in groups of maximum three hamsters per cage, with free access to water and rodent food pellets. Euthanasia was performed by carbon dioxide inhalation, and every effort was made to minimize suffering. Opisthorchis felineus metacercariae were collected from naturally infected fish (Leuciscus idus) caught in the Ob River near Novosibirsk (Western Siberia) and extracted accordingly [13].

For in vivo experiments, 83 male Syrian hamsters (aged 6-8 weeks) were chosen randomly. The animals were orally infected with 75 O. felineus metacercariae.

\section{WBC extraction from blood}

Blood samples from infected hamsters were collected by the cardiac puncture with anticoagulant (EDTA). Next, $0.3 \%$ gelatin (Sigma-Aldrich) in PBS was added to the blood samples in a 3:1 ratio, and the samples were incubated for $4-6 \mathrm{~h}$ at $37{ }^{\circ} \mathrm{C}$. This step allowed the separation of blood into an upper plasma layer containing WBCs and a lower (red-blood-cell layer). The upper layer was collected and centrifuged at $2000 \times g$ for $5 \mathrm{~min}$. The supernatant was aspirated and the pellet resuspended in PBS. This step was repeated three times. Finally, WBCs were resuspended in RPMI 1640 medium (Life Technologies, Foster City, USA) and counted.

\section{In vitro activity}

Newly excysted metacercariae (NEM) were prepared from metacercariae according to a previously published protocol [13]. Adult worms were recovered from the livers of hamsters infected three months earlier and then worms were thoroughly washed with sterile saline solution $(0.9 \% \mathrm{NaCl})$. For calculation of the half-inhibitory concentration $\left(\mathrm{IC}_{50}\right)$, we tested the following concentrations of compounds: 0.001, 0.01, 0.1, 1, 10 and $100 \mu \mathrm{M}$. The DMSO concentration across different compound concentrations was $0.5 \% \mathrm{v} / \mathrm{v}$. As control groups, we used flukes incubated in the medium with $0.5 \%$ DMSO. Thus, four to five adult worms or 30-40 NEM per well of a 12-well culture plate were analyzed. The worms were incubated at $37{ }^{\circ} \mathrm{C}$ for $24 \mathrm{~h}$ in the RPMI 1640 medium (Life Technologies) supplemented with $100 \mathrm{U} / \mathrm{ml}$ penicillin, $0.1 \mu \mathrm{g} / \mathrm{ml}$ streptomycin, $0.25 \mu \mathrm{g} / \mathrm{ml}$ amphotericin B (Sigma-Aldrich), 1\% glucose and the corresponding concentration of a drug $[13,14]$.

After $24 \mathrm{~h}$ of treatment with one of the drugs, viability of the worms was evaluated under an inverted microscope (Axiovert 40CFL, Carl Zeiss, Jena, Germany) equipped with a camera (Axiocam ICC3, Zeiss) (magnification 10$50 \times)$. The experiments were repeated three times with two replicates for each concentration. The motility of viable worms was assessed on a motility scale from 0 to $3: 3$, very active (similar movements as the control flukes); 2, active (reduced motility when compared to the control; however, the entire body still moving); 1 , reduced viability (only movements of the oral sucker); and 0, non-motile 
(non-motile for $2 \mathrm{~min}$ ) $[8,13,14]$. The $\mathrm{IC}_{50}$ value is defined as the concentration of a drug required to decrease the mean motility of a worm to $50 \%$ at the 24 -h time point. Four-parameter logistic regression was used to calculate $\mathrm{IC}_{50}$ and standard error values ( $\mathrm{R}$ package $d r c$ 3.0-1) [15]. The ANOVA lack-of-fit test was conducted to test the hypothesis that a proposed regression model fits the data well.

To assess the combinatorial activity of the anthelmintics and immune system, freshly extracted adult worms (3 months post-infection) were incubated overnight in the medium. After that, adult worms were incubated in the medium with TBN $(0.5 \mu \mathrm{M})$ or PZQ $(0.5 \mu \mathrm{M})$ and freshly extracted $4 \times 10^{5}$ WBCs for 7 days. As control groups, we used flukes incubated in the medium with $0.5 \%$ DMSO. Mortality rates were evaluated each day. The worms were classified as dead if they had a dark color and no movement was observed for 2 min [13]. To estimate mortality rates, Kaplan-Meier survival curves were built by means of the survival (v.2.38) R package. Finally, statistical difference in survival log-rank (Mantel-Haenszel test) within each pair of samples was calculated.

\section{In vivo activity}

The hamsters were subdivided into three batches for experimental treatment. In each batch, hamsters were infected with metacercariae extracted from the same batch of fish. In two batches, hamsters were treated 1 month post-infection (corresponding to infection with adult $O$. felineus worms) and 15 days post-infection (corresponding to infection with juvenile $O$. felineus). In the remaining batch, the hamsters were treated 3 months post-infection, which corresponds to chronic infection.

Groups of 5-7 hamsters were treated with a single drug dose via oral administration. PZQ and TBN was administered at $400 \mathrm{mg} / \mathrm{kg}$ body weight. Ten days after the treatment, the hamsters were placed in new cells; whole-stool samples were collected after 3 subsequent days, and $1 \mathrm{~g}$ of each stool was analyzed by means of Mini Parasep concentrators (Apacor, Wokingham, UK). The number of eggs per gram of stool (EPG) was determined. Egg reduction rates (ERR) were calculated as follows: (1 - Arithmetic Mean EPG at follow-up)/ Arithmetic Mean EPG in control $\times 100$ [10].

Worms remaining in the hepatobiliary system on day 14 post-treatment were counted after killing the hamsters by $\mathrm{CO}_{2}$ asphyxiation. Drug activity was expressed as a worm burden reduction (WBR) as described elsewhere $[8,16]$. Briefly, WBRs were calculated as follows: $(\mathrm{a}-\mathrm{b}) / \mathrm{a} \times 100$, where $\mathrm{a}$ is the average worm count in the control group upon dissection, and $\mathrm{b}$ is the average worm count in the treated group upon dissection. The significance of WBR and ERR was evaluated by the Mann-Whitney U-test and Fisher's exact test in the STATISTICA 6.0 software (Statsoft Inc, Tulsa, USA).

\section{Results \\ In vitro activity}

Evaluation of the compounds by standard motility tests against NEM revealed that $\mathrm{IC}_{50}$ of $\mathrm{TBN}\left(\mathrm{IC}_{50}=0.23 \pm\right.$ $0.059 \mu \mathrm{M}$; ANOVA lack-of-fit test: $R S S=0.02, d f=3, P=$ $0.0001)$ was similar to that of PZQ $\left(\mathrm{IC}_{50} 0.98 \pm 0.18 \mu \mathrm{M}\right.$; ANOVA lack-of-fit test: $R S S=0.008, d f=3, P=0.02$; Table 1). When the drugs were tested on adult worms, TBN effects $\left(\mathrm{IC}_{50}=0.19 \pm 0.1 \mu \mathrm{M}\right.$; ANOVA lack-of-fit test: $R S S=0.006, d f=7, P=0.0003$ ) were also similar to that of PZQ $\left(\mathrm{IC}_{50}=0.47 \pm 0.05 \mu \mathrm{M}\right.$; ANOVA lack-of-fit test: $R S S=0.009, d f=3, P=0.05$; Table 1 ).

The mortality of adult flukes was different between the PZQ and TBN treatment groups (Mantel-Haenszel log-rank test: $\left.\chi^{2}=15.8, d f=1, P<0.0001\right)$. The mortality of adult worms in the TBN group was $40 \%$ after 7 days of treatment, as compared to $80 \%$ mortality in the PZQ group after 7 days of treatment (Fig. 1a, b).

To assess if TBN and PZQ effects on liver flukes are depending on or mediated by white blood cells, adult worms were co-cultivated in the media together with WBCs freshly extracted from the blood of the infected animals and worm mortality was assessed each following day. We found that the combined action of the drug and the immune compounds caused a much greater effect on the mortality of adult helminths. In particular, the mortality of helminths in the combined group of PZQ and WBCs significantly increased (Mantel-Haenszel log-rank test: $\chi^{2}=4.8, d f=1, P=0.0289$; Fig. 1a) in comparison with the mortality of worms treated with PZQ (Fig. 1a). We observed a similar effect under the TBN treatment. Thus, the mortality of helminths treated with TBN and WBCs was significantly increased (Mantel-Haenszel log-rank test: $\chi^{2}=18.7, d f=1, P<0.0001$; Fig. 1b) when compared with the mortality in the TBN group (Fig. 1b). It should be noted that the mortality of adult flukes was not different between both combined groups PZQ + WBCs and TBN + WBCs (Mantel-Haenszel log-rank test: $\left.X^{2}=2.3, d f=1, P=0.131\right)$. WBCs had no effect on

Table $1 \mid \mathrm{I}_{50}$ values of tribendimidine and praziquantel against newly excysted metacercariae and adult Opisthorchis felineus worms

\begin{tabular}{lll}
\hline Compound & NEM & Adults \\
\hline Tribendimidine (TBN) $(\mu \mathrm{M})$ & $0.23 \pm 0.059$ & $0.19 \pm 0.1$ \\
Praziquantel $(\mathrm{PZQ})(\mu \mathrm{M})$ & $0.98 \pm 0.18$ & $0.47 \pm 0.05$
\end{tabular}

For calculation of the half-maximal inhibitory concentration $\left(\mathrm{IC}_{50}\right)$, we tested the following concentrations of compounds: $0.001,0.01,0.1,1,10$ and $100 \mu \mathrm{M}$. Data are presented as $\mathrm{IC}_{50}$ values \pm standard error (R package drc 3.0-1) Abbreviation: NEM, newly excysted metacercariae 

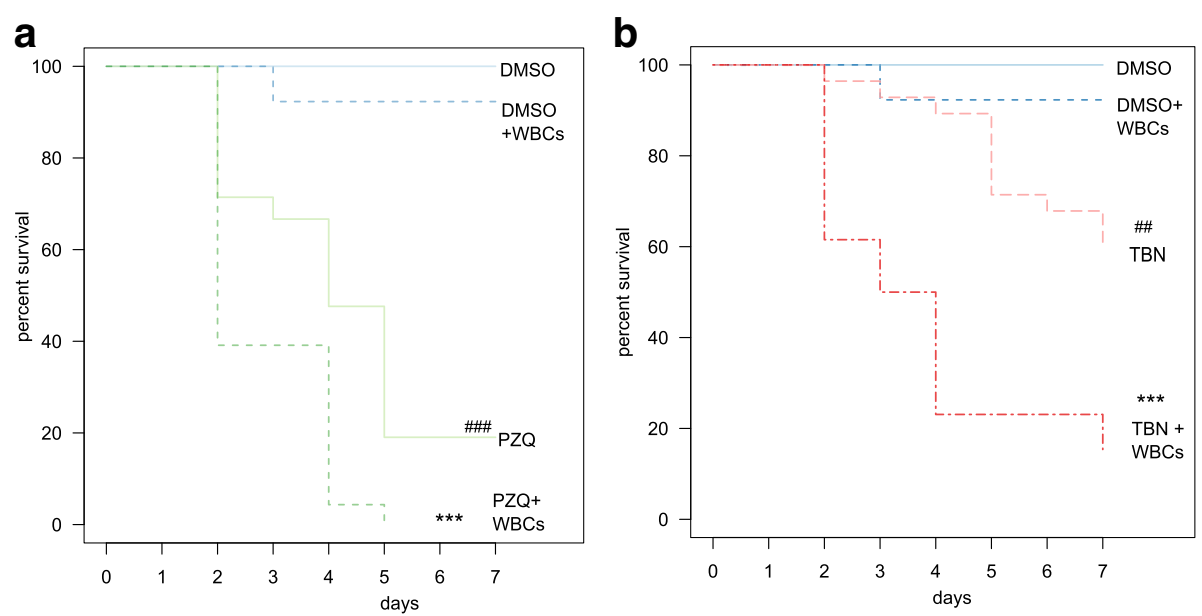

Fig. 1 Kaplan-Meier survival curves for PZQ- (a) and TBN-treated (b) adult worms. A set of Kaplan-Meier survival curves (out of three independent experiments) is shown. P-values were obtained by the survival log-rank (Mantel-Haenszel) test (R package survival v.2.38) within each pair of samples. *A significant difference between groups (treatment vs treatment + WBCs), \#A significant difference between groups (treatment vs vehicle DMSO), ${ }^{*(\# \#)} P<0.01 ;{ }^{* *(\# \#))} P<0.001$. Abbreviations: DMSO: dimethylsulfoxide; TBN: tribendimidine; PZQ: praziquantel; WBCs: white blood cells

DMSO-treated helminths (Mantel-Haenszel log-rank test: $\left.\chi^{2}=0.9, d f=1, P=0.337\right)$.

\section{In vivo activity}

We assessed the effect of TBN at different stages of the disease, specifically 15-day and 1-month infections, which are considered acute opisthorchiasis, and 3 month chronic opisthorchiasis. The treatment of hamsters 15 days post-infection with TBN at $400 \mathrm{mg} / \mathrm{kg}$ resulted in a WBR of $90.7 \%$ (Mann-Whitney U-test: $U=0, d f=4, P=$ 0.009; Table 2). The treatment of hamsters 1 month post-infection with TBN at $400 \mathrm{mg} / \mathrm{kg}$ caused a WBR of 90\% (Mann-Whitney U-test: $U=0, d f=4, P=0.009$; Table 2).
Treatment with PZQ at $400 \mathrm{mg} / \mathrm{kg} 1$ month post-infection resulted in a WBR of $76.5 \%$ (Mann-Whitney U-test: $U$ $=0, d f=6, P=0.002)$. According to Fisher's exact test $(P=$ 0.278 ; 0.447), the differences between WBR values after PZQ and TBN treatment 1 month post-infection were not significant.

Treatment of hamsters with chronic infection (3 months post-infection) with TBN at $400 \mathrm{mg} / \mathrm{kg}$ caused a WBR of 76\% (Mann-Whitney U-test: $U=0, d f=4, P=0.009$; Table 2). For comparison, treatment of hamsters with chronic infection with PZQ resulted in a WBR of $77.2 \%$ (Mann-Whitney U-test: $U=0, d f=10, P=0.014$; Table 2). The differences in worm reduction rates between acute and chronic infection for TBN were not significant (Fisher's exact test: $P=0.305$ ).

Table 2 Worm burden reduction values obtained after treatment of hamsters harboring $O$. felineus with tribendimidine or praziquantel

\begin{tabular}{|c|c|c|c|c|c|}
\hline & Number of animals & Mean no. of worms $\pm S D$ & WBR (\%) & Mean EPG \pm SD & ERR (\%) \\
\hline \multicolumn{6}{|c|}{ Treatment of chronic infection (3 months) } \\
\hline Control & 13 & $33 \pm 10$ & - & $1863 \pm 1006$ & - \\
\hline TBN, 400 mg/kg & 5 & $7 \pm 4$ & 76 & $927 \pm 620$ & 51.0 \\
\hline PZQ, 400 mg/kg & 11 & $8 \pm 5$ & 77.2 & nd & nd \\
\hline \multicolumn{6}{|c|}{ Treatment of acute infection (1 month) } \\
\hline Control $^{1}$ & 5 & $19 \pm 2$ & - & $863 \pm 279$ & - \\
\hline Control $^{2}$ & 6 & $34 \pm 9$ & - & nd & - \\
\hline $\mathrm{TBN}^{1}, 400 \mathrm{mg} / \mathrm{kg}$ & 5 & $2 \pm 4$ & 90 & $299 \pm 244$ & 65.4 \\
\hline $\mathrm{PZQ}^{2}, 400 \mathrm{mg} / \mathrm{kg}$ & 7 & $8 \pm 6$ & 76.5 & nd & nd \\
\hline \multicolumn{6}{|c|}{ Treatment of acute infection (15 days) } \\
\hline $\mathrm{TBN}^{1}, 400 \mathrm{mg} / \mathrm{kg}$ & 5 & $2 \pm 2$ & 90.7 & $463 \pm 281$ & 46.3 \\
\hline
\end{tabular}

Superscript numbers denote data relative to the corresponding control group Abbreviations: TBN, tribendimidine; PZQ, praziquantel; SD, standard deviation, WBR, worm burden reduction rate, EPG, eggs per gram of feces, ERR, egg reduction rates, nd, not determined 


\section{Discussion}

In this study, we compared the anthelmintic activity of TBN and PZQ on the experimental model of the liver fluke infection caused by O. felineus. Our data indicate that the efficacy of TBN is similar to that of PZQ in vitro and in vivo on different stages of the disease: at one and three months after the infection.

TBN efficacy against $O$. felineus are in compliance with the data shown on O. viverrini $[8-10]$. The $\mathrm{IC}_{50}$ for TBN in vitro against adult $O$. viverrini was shown to be $0.05 \mu \mathrm{g} / \mathrm{ml}$ [8]. A similar efficacy of a single dose in vivo has been shown in studies against O. viverrini: $400 \mathrm{mg} /$ $\mathrm{kg}$ of TBN treatment was sufficient for 95.7\% WBR in hamsters four weeks after the infection [9]. High efficacy of TBN has also been demonstrated on an experimental model of clonorchiasis (4-6 weeks of C. sinensis infection): a $150 \mathrm{mg} / \mathrm{kg}$ dose was sufficient for $91.5 \% \mathrm{WBR}$, and $240 \mathrm{mg} / \mathrm{kg}$ resulted in $99.4 \%$ WBR [17].

High cure rates were observed with TBN (90\%) and PZQ (90\%) among O. viverrini-infected participants with low-intensity infections [10]; however, several studies have shown lower efficacy rates for TBN and PZQ in the treatment of moderate and heavy infections (44\% for TBN and 56\% for PZQ) [12, 18]. Thus, TBN has efficacy comparable to that of $\mathrm{PZQ}$ in the treatment of liver fluke infections. Nevertheless, TBN was shown to have a good safety profile and caused fewer adverse events [10] compared with PZQ when tested on participants with $O$. viverrini infection. In particular, in one clinical trial, participants treated with PZQ were about four times more likely to have an adverse event than those treated with TBN [10]. All adverse events in the TBN group were mild except for nausea. Moderate adverse events were reported in the PZQ group, including vertigo, nausea, fatigue, abdominal cramps and vomiting [10].

We also demonstrated that apparently there is an immunity-dependent lethal effect of TBN on the liver flukes O. felineus. The mortality of helminths in the combined group of TBN and WBCs significantly increased in contrast to that of worms treated with TBN alone.

Although both substances have completely different chemical structures (suggesting that they must act on different molecular targets), they have similar effects on the appearance of helminths. In particular, both drugs cause helminth immobilization and paralysis and damage the tegument $[17,19]$. TBN acts as a cholinergic agonist of the B subtype and L subtype of nicotinic acetylcholine receptors [11]. In contrast, the precise mechanism of action of PZQ has not yet been elucidated (reviewed in [20]). Exposure of worms to PZQ causes a massive influx of calcium, contraction of the musculature, disruption of the tegument, and effects on calcium channels (reviewed in [20]). The disruption of the tegument presumably allows immune cells of the host to attack the parasite's tissues and organs (reviewed in [20]). Accordingly, simultaneous administration of WBCs with PZQ in our study resulted in a higher mortality rate than that of PZQ alone (Fig. 1a). These findings are in agreement with the data on schistosomes. It has been reported that immune effector mechanisms may be synergistically involved in the action of PZQ on worms [21]. In particular, administration of rabbit anti-Schistosoma mansoni antisera simultaneously with PZQ yielded a greater WBR relative to PZQ alone.

The tegument of trematodes is a multinuclear syncytium that is approximately $4 \mu \mathrm{m}$ thick and has several vital functions, including protection from immune cells of the host, absorption of nutrients, ion transport and communication with the underlying nervous system. The tegument is in direct contact with muscle fibers, ensuring an instantaneous reaction of the muscles to external stimuli, such as mechanical pressure, an ion gradient, or a gradient of nutrients [22]. Furthermore, the ability to damage the tegument has been described for other anthelmintics, such as artemether [17], artesunate [23] and mefloquine [24]. It should be noted that many anthelmintic agents act on different molecular targets but have the same effect: tegumental damage. This fact does not mean a direct effect of the drugs on a molecular target within the tegument but rather an indirect effect through disturbances in the physiology of helminths and damage to the parasite's tissues and organs.

Mortality in vitro in combined groups of adult worms treated with $\mathrm{TBN}+\mathrm{WBCs}$ and $\mathrm{PZQ}+\mathrm{WBCs}$ was almost the same. These findings are in agreement with the results from our in vivo study, where respective single oral doses of TBN and PZQ resulted in similar anthelmintic effects.

\section{Conclusions}

For decades, treatment of the liver fluke infection caused by Opisthorchis felineus has been based on PZQ. Our findings on an experimental model showed that TBN is also an efficient drug for therapy of this pathology. Both in vivo and in vitro experiments demonstrated its high efficacy against $O$. felineus. Hence, it can be a potential candidate for the treatment of the disease.

\footnotetext{
Abbreviations

DMSO: Dimethylsulfoxide; EPG: Eggs per gram of feces; ERR: Egg reduction rate; $I_{50}$ : Half-inhibitory concentration; PZQ: Praziquantel;
}

TBN: Tribendimidine; WBCs: White blood cells; WBR: Worm burden reduction

\section{Acknowledgments}

We acknowledge the support and collaboration of members of the TOPIC (Tomsk OPIsthorchiasis Consortium). Fluorescence microscopy was performed at the Microscopy Center of the Institute of Cytology and Genetics, the Siberian Branch of the Russian Academy of Sciences (ICG SB RAS). The English language was corrected by Shevchuk Editing. 


\section{Funding}

This work was supported financially by the Russian Science Foundation (grant number 18-15-00098). The funding agency had no role in this study, e.g. in study design, data collection, or decision to publish.

\section{Availability of data and materials}

All data generated or analyzed during this study are included in this published article.

\section{Authors' contributions}

VAS synthesized tribendimidine and confirmed its purity. MYP developed the study protocol and analysis of the results. EAS was responsible for animal work. VAM provided study oversight. All authors participated in drafting the manuscript, contributed to its revisions and approved the final manuscript.

\section{Ethics approval}

All procedures were in compliance with EU Directive 2010/63/EU for anima experiments. The animals were kept and treated according to the protocols approved by the Committee on the Ethics of Animal Experiments of the Institute of Cytology and Genetics of the Siberian Branch of the Russian Academy of Sciences (ICG SB RAS; permit number: 42 of 25.05.2018).

\section{Consent for publication}

Not applicable.

\section{Competing interests}

The authors declare that they have no competing interests.

\section{Publisher's Note}

Springer Nature remains neutral with regard to jurisdictional claims in published maps and institutional affiliations.

\section{Author details}

${ }^{1}$ Institute of Cytology and Genetics of the Siberian Branch of the Russian Academy of Sciences (SB RAS), 10 Lavrentiev Ave, Novosibirsk 630090, Russia. ${ }^{2}$ Novosibirsk State University, 2 Pirogova Str, Novosibirsk 630090, Russia. ${ }^{3}$ Vorozhtsov Institute of Organic Chemistry of SB RAS, Novosibirsk, Russia. ${ }^{4}$ Institute of Systematics and Ecology of Animals, SB RAS, Novosibirsk, Russia.

Received: 4 October 2018 Accepted: 3 January 2019

Published online: 11 January 2019

\section{References}

1. Be'er SA. Biology of the agent of opisthorchiasis. Moscow: KMK Scientific Press Ltd; 2005.

2. Pakharukova MY, Mordvinov VA. The liver fluke Opisthorchis felineus: biology, epidemiology, and carcinogenic potential. Trans R Soc Trop Med Hyg. 2016; 110:28-36.

3. Pozio E, Armignacco O, Ferri F, Gomez Morales MA. Opisthorchis felineus, an emerging infection in Italy and its implication for the European Union. Acta Trop. 2013;126:54-62.

4. IARC, IARC working group on the evaluation of carcinogenic risks to humans. Biological agents. A review of human carcinogens. In: IARC Monographs on the Evaluation of Carcinogenic Risks to Humans. 2012. 100:1-441.

5. Maksimova GA, Pakharukova MY, Kashina EV, Zhukova NA, Kovner AV, Lvova MN, et al. Effect of Opisthorchis felineus infection and dimethylnitrosamine administration on the induction of cholangiocarcinoma in Syrian hamsters. Parasitol Int. 2017;66:458-63.

6. Gouveia MJ, Pakharukova MY, Laha T, Sripa B, Maksimova GA, Rinaldi G, et al. Infection with Opisthorchis felineus induces intraepithelial neoplasia of the biliary tract in a rodent model. Carcinogenesis. 2017;38:929-37.

7. Prichard RK, Basanez MG, Boatin BA, McCarthy JS, Garcia HH, Yang GJ, et al. A research agenda for helminth diseases of humans: intervention for control and elimination. PLoS Negl Trop Dis. 2012;6:e1549.

8. Keiser J, Adelfio R, Vargas M, Odermatt P, Tesana S. Activity of tribendimidine and praziquantel combination therapy against the liver fluke Opisthorchis viverrini in vitro and in vivo. J Helminthol. 2013;87:252-6.

9. Keiser J, Shu-Hua X, Chollet J, Tanner M, Utzinger J. Evaluation of the in vivo activity of tribendimidine against Schistosoma mansoni, Fasciola hepatica, Clonorchis sinensis, and Opisthorchis viverrini. Antimicrob Agents Chemother. 2007;51:1096-8
10. Sayasone S, Keiser J, Meister I, Vonghachack Y, Xayavong S, Senggnam K, et al. Efficacy and safety of tribendimidine versus praziquantel against Opisthorchis viverrini in Laos: an open-label, randomised, non-inferiority, phase 2 trial. Lancet Infect Dis. 2018;18:155-61.

11. Robertson AP, Puttachary S, Buxton SK, Martin RJ. Tribendimidine: mode of action and nAChR subtype selectivity in Ascaris and Oesophagostomum. PLoS Negl Trop Dis. 2015;9:e0003495.

12. Sripa B. Tribendimidine: an alternative to praziquantel to treat human liver fluke infection? Lancet Infect Dis. 2018;18:124-5.

13. Pakharukova MY, Shilov AG, Pirozhkova DS, Katokhin AV, Mordvinov VA. The first comprehensive study of praziquantel effects in vivo and in vitro on European liver fluke Opisthorchis felineus (Trematoda). Int J Antimicrob Agents. 2015;46:94-100.

14. Mordvinov VA, Shilov AG, Pakharukova MY. Anthelmintic activity of cytochrome P450 inhibitors miconazole and clotrimazole: in vitro effect on the liver fluke Opisthorchis felineus. Int J Antimicrob Agents. 2017;50:97-100.

15. Ritz C, Baty F, Streibig JC, Gerhard D. Dose-response analysis using R. PLoS One. 2015;10:e0146021.

16. Pakharukova MY, Pakharukov Y , Mordvinov VA. Effects of miconazole/ clotrimazole and praziquantel combinations against the liver fluke Opisthorchis felineus in vivo and in vitro. Parasitol Res. 2018;117:2327-31.

17. Xiao SH, Keiser J, Xue J, Tanner M, Morson G, Utzinger J. Effect of singledose oral artemether and tribendimidine on the tegument of adult Clonorchis sinensis in rats. Parasitol Res. 2009;104:533-41.

18. Qian MB, Yap P, Yang YC, Liang H, Jiang ZH, Li W, et al. Efficacy and safety of tribendimidine against Clonorchis sinensis. Clin Infect Dis. 2013;56:e76-82.

19. Keiser J, Utzinger J, Xiao SH, Odermatt P, Tesana S. Opisthorchis viverrini: efficacy and tegumental alterations following administration of tribendimidine in vivo and in vitro. Parasitol Res. 2008;102:771-6.

20. Day TA, Botros S. Drug resistance in schistosomes. In: Maule AG, Marks NJ, editors. Parasitic flatworms: Molecular Biology, Biochemistry, Immunology and Physiology. Wallingford: CABl; 2006. p. 256-65.

21. Doenhoff MJ, Sabah AA, Fletcher C, Webbe G, Bain J. Evidence for an immune-dependent action of praziquantel on Schistosoma mansoni in mice. Trans R Soc Trop Med Hyg. 1987;81:947-51.

22. Thompson DP, Geary TG. Helminth surfaces: structural, molecular and functional properties. In: Marr JJ, Nilsen TW, Komuniecki RW, editors. Molecular Medical Parasitology. London: Academic Press; 2003. p. 298-38.

23. Keiser J, Vargas M. Effect of artemether, artesunate, OZ78, praziquantel, and tribendimidine alone or in combination chemotherapy on the tegument of Clonorchis sinensis. Parasitol Int. 2010;59:472-6.

24. Xiao SH, Xue J, Shen BG. Tegumental alterations of adult Schistosoma japonicum harbored in mice treated with a single oral dose of mefloquine. Zhongguo Ji Sheng Chong Xue Yu Ji Sheng Chong Bing Za Zhi. 2010;28:1-7.

Ready to submit your research? Choose BMC and benefit from:

- fast, convenient online submission

- thorough peer review by experienced researchers in your field

- rapid publication on acceptance

- support for research data, including large and complex data types

- gold Open Access which fosters wider collaboration and increased citations

- maximum visibility for your research: over $100 \mathrm{M}$ website views per year

At $\mathrm{BMC}$, research is always in progress.

Learn more biomedcentral.com/submissions 